\title{
Subjective prosthodontic treatment need, tooth loss and associated factors among dental patients in Dar es salaam, Tanzania
}

\author{
Lorna C Carneiro ${ }^{1}$, Silas Sembikoํㅜ Joyce R Masalu²
}

1. Department of Restorative Dentistry, School of Dentistry, Muhimbili University of Health and Allied Sciences, P. O. Box 65451, Dar es salaam, Tanzania.

2. Department of Orthodontic, Pedodontic and Community Dentistry, School of Dentistry, Muhimbili University of Health and Allied Sciences, P. O. Box 65014, Dar es salaam, Tanzania.

\section{Co-author Emails:}

Lorna C Carneiro: carneiro2@hotmail.com; Silas Sembiko: silas.sembiko@gmail.com; Joyce R Masalu: rfumbuka@yahoo.com

\begin{abstract}
Background: A full complement of teeth has been shown to be a prerequisite for a healthy masticatory system and satisfactory function and having tooth loss can be undesirable.

Objective: To determine the subjective prosthodontic treatment need, tooth loss and associated factors among patients attending dental clinics in Dar-es-salaam, Tanzania.

Methods: This cross-sectional study was conducted among subjects with tooth loss aged 18 years and above attending public dental clinics in Dar-es-Salaam, Tanzania. A questionnaire and clinical examination were used to obtain data. Chi-Square test and logistic regression analyses were performed and a p-value of $\leq 0.05$ was considered to be statistically significant.

Results: Majority of the 402 participants were aged 18-34 years (52.2\%), females (64.9\%) and with primary level of education or less $(52.2 \%)$. Subjective prosthodontic treatment need was indicated by more than half of the participants $54.2 \%$ ) and those who lost 4 or more teeth and those who lost teeth upper anterior had higher odds of expressing subjective treatment need (OR=2.6; $\mathrm{CI}=1.5-4.3 \& \mathrm{OR}=4.9 ; \mathrm{CI}=2.2-10.8$ respectively).

Conclusions: This study highlights that having four or more missing teeth and having tooth loss in the anterior location of the upper jaw were significant contributing factors in expressing patient's subjective prosthodontic treatment need.

Keywords: Subjective prosthodontic treatment need, tooth loss; dental patients; Dar es salaam; Tanzania.

DOI: bttps://dx.doi.org/10.4314/abs.v21i4.49

Cite as: Carneiro LC, Sembiko S, Masalu JR. Subjective prosthodontic treatment need, tooth loss and associated factors among dental patients in Dar es salaam, Tanzania. Afri Health Sci. 2021;21(4):1905-13. https:// dx.doi.org/10.4314/abs.v21i4.49
\end{abstract}

\section{Introduction}

A full complement of teeth is a prerequisite for a healthy masticatory system, satisfactory function ${ }^{1}$ and aesthetics ${ }^{2}$. Having tooth loss is undesirable irrespective of the cause $\mathrm{e}^{3}$ and tooth retention profile of populations is influenced not only by dental diseases but also by socio-economic, behavioral and attitudinal characteristics ${ }^{4}$.

\section{Corresponding author:}

Lorna C Carneiro,

P. O. Box 65451, Dar es salaam, Tanzania.

Telephone number: +255713835140

Email: carneiro2@hotmail.com
Subjective prosthodontic treatment need is determined by functional, esthetic, psychological and social impacts due to tooth $\operatorname{loss}^{5}$. Different levels of prosthodontic interventions are needed as a standard of care to improve conditions of persons with tooth loss ${ }^{6}$. Nevertheless not all patients with incomplete dental arch need treatment or perceive their condition as harmful or deleterious?

In Tanzania, tooth loss is a common finding ${ }^{8}$ with reported objective need for tooth replacement being higher among patients having missing teeth in both anterior and posterior regions 9 . However, there is paucity of information on the subjective prosthodontic treatment need of patients in relation to number and location of missing teeth. This study therefore aimed to determine 
the subjective prosthodontic treatment need, tooth loss and associated factors among patients attending public dental clinics in Dar es Salaam, Tanzania. The results of this study will provide baseline information for comparative studies regarding the subjective prosthodontic treatment need of Tanzanians, the pattern of tooth loss and associated factors. Furthermore, it will assist in the planning of preventive strategies and objective need for prosthodontic treatment.

\section{Methods}

This cross-sectional hospital-based study involved dental patients aged 18 years old and above who attended public dental clinics in Dar es Salaam, Tanzania. Dar es salaam was purposively selected due to the population density from all over Tanzania ${ }^{10}$ and because of the likelihood of getting many patients in the clinics. An urban and rural representation was obtained by selection of one public dental clinic from each of the three municipalities (Kinondoni, Ilala and Temeke) in Dar es salaam. The consultant referral dental clinic at the Muhimbili National Hospital was further selected as it receives referred patients from all over the country. At each hospital, dental patients aged 18 years old and above who attended the selected dental clinics during the study period were conveniently selected so as to obtain the estimated sample size of 422 .

Enrolled in the study were attending patients aged 18 years and above with one or more missing teeth. Subjects excluded from the study were patients who did not have tooth loss, those mentally challenged/handicap, presenting with emergency dental conditions or oral tumors/cancers and those with removable or fixed prosthesis.

Ethical clearance was granted by the Muhimbili University of Health and Allied Sciences (MUHAS) while permission to conduct the research in respective dental clinics was obtained from the respective administrative authorities. Prior to obtaining individual written consent patients awaiting dental treatment at each clinic were informed of the study objectives and assured of their confidentiality and the right to participate or withdraw from the study without penalty.

Data were collected from the study participants through an interview using a structured questionnaire followed by a clinical examination. Following consent subjects were seated comfortably in the dental chair and interviewed by one of the investigators (SS) using a structured questionnaire. The first section of the ques- tionnaire obtained information with regards to socio demographic characteristics. Participants were categorized by age into two groups 18-34 years $=0$ and 35-85 years $=1$ based on the median age of 34 years while their sex was recorded as male $=0$ or female $=1$. The level of education of participants was recorded as having "no formal education, primary education, secondary education and college/university education" which were then dichotomized into primary education or less $=0$ and secondary education or more $=1$.

The second section of the questionnaire determined if a subject had missing teeth based on no $=0$ and yes $=1$ response and those with missing teeth reported their subjective prosthodontic treatment need using a yes $=$ 0 or no $=1$ response.

Clinical examination of subject's oral cavity was then performed by the same investigator using a mouth mirror and illumination from the overhead light. Findings were recorded in a clinical survey form that had a chart of all the 32 teeth. Teeth that were present in the oral cavity and with more than half of its crown intact were scored $=0$ while teeth that were missing or with more than half of its crown absent were scored $=1$.

Subjects were further grouped according to number of missing teeth $(1-3=0$ and 4 or more $=1)$ and location of missing tooth/teeth (tooth number 13-23 = upper anterior jaw; tooth number 14-18 or 24-28 = upper posterior jaw; tooth number 33-43 = lower anterior jaw and tooth 34-38 or 44-48 = lower posterior jaw). Intra-examiner consistency was assessed daily by randomly selecting $10 \%$ of patients enrolled in the study. Kappa value of 0.87 was obtained.

To assess for clarity and to ensure that the questionaire measured what it was supposed to measure and that the clinical record form was usable, a field testing was conducted on 40 conveniently selected patients attending the dental clinic of Muhimbili National Hospital. Test-retest for assessing reliability of the questionnaire was not done because it was not possible to invite participants for a second administration of the questionnaire. Calibration of examiner SS with respect to clinical assessment was carried out against LCC.

The data were processed and analyzed using SPSS software for Windows version 20. Cross-tabulations and Chi-Square test was used to determine bivariate associations with $\mathrm{p}$-value $\leq 0.05$ being considered to be statistically significant. Multiple logistic regression of dependent variable and independent variables was performed to control for confounding effects. 


\section{Results}

A total of 402 dental patients with one or more missing teeth participated in the study giving a response rate of $95.3 \%$. Age ranged from $18-85$ years with mean age of $36.16 \pm 13.246$ years and median age of 34 years. The majority of subjects were from age group 18-34 years $(52.2 \%)$, females $(64.9 \%)$ and having primary or less level of education (52.2\%). Many more participants had three or less missing teeth $(56.5 \%)$ with many more missing teeth in the lower posterior jaw (84.6\%), upper posterior jaw (66.4\%), upper anterior jaw (16.7\%) and lower anterior jaw $(4.5 \%)$ respectively. Subjective prosthodontic treatment need was expressed by $54.2 \%$ of the participants (Table 1).

Table 1: Distribution of participants by sociodemographic factors, missing teeth and subjective treatment need, $(\mathrm{N}=402)$.

\begin{tabular}{|llll|}
\hline Variables & & $\mathrm{n}$ & $\%$ \\
Age Group (years) & $18-34$ & 210 & 52.2 \\
& $35-85$ & 192 & 47.8 \\
Level of Education & Male & 141 & 35.1 \\
& Female & 261 & 64.9 \\
Number of missing teeth & Primary School or less & 210 & 52.2 \\
& Secondary School or more & 192 & 47.8 \\
Location of missing teeth & Upper jaw - Anterior & 227 & 56.5 \\
& Upper jaw - Posterior & 175 & 43.5 \\
& Lower jaw - Anterior & 18 & 16.7 \\
& Lower jaw - Posterior & 340 & 66.4 \\
\multirow{2}{*}{ Subjective treatment } & No & 184 & 4.5 \\
need & & & 45.8 \\
& Yes & 218 & 54.2 \\
\hline
\end{tabular}

Table 2 shows the distribution of number and location of missing teeth of participants by socio-demographic characteristics. A statistically significant higher proportion of those who were in age group 35-85 years $(67.4 \%)$, females $(70.3 \%)$ and those with primary education or less $(60.0 \%)$ had 4 or more missing teeth in comparison to their counterparts. In regards to location of missing teeth, a significantly higher proportion of participants in 35-85 years had missing teeth in the anterior area of the upper jaw (74.6\%) with no significant differences observed between males and females or between the different levels of education. Having more missing teeth in the posterior area of the upper jaw was significantly linked to being of age group 35-85 years age group $(56.9 \%)$ and having primary or less education (56.9\%). There was no significant association between different sexes and missing teeth in upper posterior location. In the lower jaw, the observed statistically significant difference between those aged 35 and above who had missing teeth in the anterior area as compared to none in age less than 35 years is noted. A significantly higher number of those aged 35 and above who had missing teeth in the anterior area were having primary or less education $(88.9 \%)$. Having missing teeth in the posterior area of lower jaw showed no association with age, sex or level of education. 
Table 2: Distribution of participants by missing teeth and socio-demographic characteristics.

\begin{tabular}{|c|c|c|c|c|c|c|c|}
\hline \multirow[b]{3}{*}{ Missing Teeth } & \multicolumn{6}{|c|}{ Demographic characteristics } & \multirow{4}{*}{ Total } \\
\hline & \multicolumn{2}{|c|}{ Age Group (years) } & \multicolumn{2}{|c|}{ Sex } & \multicolumn{2}{|c|}{ Level of education } & \\
\hline & $18-34$ & $35-85$ & Male & Female & Primary & Secondary & \\
\hline & & & & & or less & or more & \\
\hline \multicolumn{8}{|c|}{ Number of missing teeth } \\
\hline 3 or less & $153(67.4)$ & $74(32.6)$ & $89(39.2)$ & $138(60.8)$ & $105(46.3)$ & $122(53.7)$ & 227 \\
\hline 4 or more & $57(32.6)$ & $118(67.4)^{* * *}$ & $52(29.7)$ & $123(70.3)^{*}$ & $105(60.0) * *$ & $70(40.0)$ & 175 \\
\hline \multicolumn{8}{|c|}{ Location of missing teeth } \\
\hline \multicolumn{8}{|c|}{ Upper jaw - anterior } \\
\hline No & $193(57.6)$ & $142(42.4)$ & $116(34.6)$ & $219(65.4)$ & $172(51.3)$ & $163(48.7)$ & 335 \\
\hline Yes & $17(25.4)$ & $50(74.6)^{* * *}$ & $25(37.3)$ & $42(62.7)$ & $38(56.7)$ & $29(43.3)$ & 67 \\
\hline \multicolumn{8}{|c|}{ Upper jaw - posterior } \\
\hline No & $95(70.4)$ & $40(29.6)$ & $53(39.3)$ & $82(60.7)$ & $58(43.0)$ & $77(57.0)$ & 135 \\
\hline Yes & $115(43.1)$ & $152(56.9)^{* * *}$ & $88(33.0)$ & $179(67.0)$ & $152(56.9) * *$ & $115(43.1)$ & 267 \\
\hline \multicolumn{8}{|c|}{ Lower jaw - anterior } \\
\hline No & $210(54.7)$ & $174(45.3)$ & $134(34.9)$ & $250(65.1)$ & $194(50.5)$ & $190(49.5)$ & 348 \\
\hline Yes & $0(0.0)$ & $18(100)^{* * *}$ & $7(38.9)$ & $11(61.1)$ & $16(88.9) * * *$ & $2(11.1)$ & 18 \\
\hline \multicolumn{8}{|c|}{ Lower jaw - posterior } \\
\hline No & $33(53.2)$ & $29(46.8)$ & $26(41.9)$ & $36(58.1)$ & $26(41.9)$ & $36(58.1)$ & 62 \\
\hline Yes & $177(52.1)$ & $163(47.9)$ & $115(33.8)$ & $225(66.2)$ & $184(54.1)$ & $156(45.9)$ & 380 \\
\hline
\end{tabular}

The distribution of subjective prosthodontic treatment need of participants by socio-demographic characteristics is shown in Table 3. Statistically significantly higher proportion $(61.5 \%)$ of participants aged $35-85$ years expressed subjective prosthodontic treatment need in comparison to those aged $18-34$ years $(47.6 \%)$. The subjective prosthodontic treatment need of participants did not vary across sexes or education levels.

Table 3: Distribution of subjective prosthodontic treatment need of participants by sociodemographic characteristics.

\begin{tabular}{|c|c|c|c|c|}
\hline & & \multicolumn{3}{|c|}{ Subjective prosthodontic treatment need } \\
\hline \multicolumn{2}{|c|}{ Socio demographic characteristics } & No & Yes & Total \\
\hline \multirow[t]{2}{*}{ Age (years) } & $18-34$ & $110(52.4)$ & $100(47.6)$ & $210 * *$ \\
\hline & $35-85$ & $74(38.5)$ & $118(61.5)$ & 192 \\
\hline \multirow[t]{2}{*}{$\operatorname{Sex}$} & Male & $66(46.8)$ & $75(53.2)$ & $141^{\mathrm{ns}}$ \\
\hline & Female & $118(45.2)$ & $143(54.8)$ & 261 \\
\hline \multirow{2}{*}{$\begin{array}{l}\text { Level of } \\
\text { education }\end{array}$} & Primary or less & $100(47.6)$ & $110(52.4)$ & $210^{\mathrm{ns}}$ \\
\hline & Secondary or more & $84(43.8)$ & $108(56.2)$ & 192 \\
\hline
\end{tabular}


Shown in Table 4 is the distribution of subjective prosthodontic treatment need of participants by number and location of missing teeth. The subjective prosthodontic treatment need of participants who were missing 4 or more teeth $(70.3 \%)$ was statistically significantly higher than those who were missing 3 or less $(41.9 \%)$. The subjective prosthodontic treatment need was statistical- ly significantly higher among participants with missing teeth in the anterior location of the upper jaw $(86.6 \%)$, posterior location of the upper jaw (58.4\%) and anterior location of lower jaw $(77.8 \%)$. There was no statistically significant difference in subjective treatment need between participants having or not having missing teeth in the posterior location of lower jaw.

Table 4. Distribution of subjective prosthodontic treatment need of participants by number and location of missing teeth

\begin{tabular}{|lllll|}
\hline \multicolumn{5}{|c}{ Subjective prosthodontic treatment need } \\
Missing teeth & No & Yes & Total \\
Number of missing teeth & & & \\
& 3 or less & $132(58.1)$ & $95(41.9)$ & $227^{* *}$ \\
& 4 or more & $52(29.7)$ & $123(70.3)$ & 175 \\
Location of missing teeth & & & \\
Upper jaw & No & $175(52.2)$ & $160(47.8)$ & $335^{* *}$ \\
-anterior & Yes & $9(13.4)$ & $58(86.6)$ & 67 \\
Upper jaw & No & $73(54.1)$ & $62(45.9)$ & $135^{*}$ \\
-posterior & Yes & $111(41.6)$ & $156(58.4)$ & 267 \\
Lower jaw & No & $180(46.9)$ & $204(53.1)$ & $384^{*}$ \\
-anterior & Yes & $4(22.2)$ & $14(77.8)$ & 18 \\
Lower jaw & No & $30(48.4)$ & $32(51.6)$ & $62^{\text {ns }}$ \\
-posterior & Yes & $154(45.3)$ & $186(54.7)$ & 340 \\
\hline ns =Not significant; $p \leq 0.01^{* *}, p \leq 0.05 *$ & & \\
\hline
\end{tabular}

Table 5 shows adjusted odds ratios (ORs) and 95\% confidence intervals (CIs). To determine the likelihood of expressing subjective prosthodontic need; independent variables including age, number of missing upper anterior, upper posterior and lower anterior location teeth were regressed upon subjective prosthodontic treatment need as a dependent variable.
Those with 4 or more missing teeth $(\mathrm{OR}=2.6$; $\mathrm{CI}=1.56$ 4.39) and those with missing teeth in the upper anterior location ( $\mathrm{OR}=4.97 ; \mathrm{CI}=2.27-10.86)$ had higher odds of expressing subjective prosthodontic treatment need. Nagelkerke R2 $=0.172 \approx 17 \%$. 
Table 5. Odds ratios (ORs) and 95\% confidence intervals (CIs) for subjective prosthodontic treatment need according to age, number of missing teeth, teeth missing in upper anterior, upper posterior and lower anterior location.

\begin{tabular}{|llll|}
\hline & p-value & OR & $95 \%$ CI \\
Age & & & \\
18 -34yrs vs. $\geq 35$ yrs & 0.711 & 1.090 & $0.692-1.716$ \\
Number of missing teeth & 0.001 & 2.613 & $1.557-4.386$ \\
$\leq 3$ teeth vs. $\geq 4$ teeth & & & \\
$\begin{array}{l}\text { Teeth missing in upper } \\
\text { jaw -anterior area }\end{array}$ & & & \\
Yes vs. No & 0.001 & 4.968 & $2.272-10.862$ \\
$\begin{array}{l}\text { Teeth missing in upper } \\
\text { jaw - posterior area }\end{array}$ & & & \\
Yes vs. No & 0.607 & 0.874 & $0.523-1.460$ \\
$\begin{array}{l}\text { Teeth missing in lower } \\
\text { jaw - anterior area }\end{array}$ & & & \\
Yes vs. No & 0.918 & 0.932 & $0.247-3.514$ \\
\hline
\end{tabular}

\section{Discussion}

This hospital based cross-sectional study assessed the subjective prosthodontic treatment need of patients aged 18-85 years attending selected public dental clinics in Dar es Salaam, Tanzania. The involvement of public dental clinics was based on expectation of getting a wide coverage of Tanzanians with missing teeth from both urban and rural populations. Limitation of the sampling methods used and possible recall bias with regards to the questionaire were considered. However, the results provide an insight on the expressed subjective prosthodontic treatment need of Tanzanian patients and the influence of socio demographic characteristics, number and location of missing teeth. The data obtained from this study can be used for comparison with other studies within and outside the country.

This study had a higher number of subjects within the age group of 35-72 years with 4 or more missing teeth than their younger counterparts. Similar findings were reported in studies done in Asia ${ }^{11}$, Brazil ${ }^{12}$ and Europe $^{13}$. On the contrary, a study done in Uganda ${ }^{14}$ reported young adults to have lost more teeth. The higher number of missing teeth amongst the older age group in this study could be related to inadequate oral hygiene practices and possibly the cumulative effect of oral diseases ${ }^{15}$. In addition barriers to seeking oral health care experienced by many in the past could have led to emergency oral care services in form of tooth extraction ${ }^{16}$. Unlike other studies ${ }^{17}$ where males had more missing teeth, this study observed a higher number of missing teeth in females. Similar finding have been reported in another study done in Tanzania ${ }^{8}$ and other studies in Uganda ${ }^{14}$, Sudan ${ }^{18}$ and Nigeria ${ }^{19}$. Higher number of missing teeth reported among females in this study could be attributed to their behavior of snacking and that of visiting the dentist more frequently ${ }^{20}$.

The higher number of missing teeth amongst those with lower level of education in this study was similarly reported in other studies ${ }^{21,22}$. Contrastingly a study done in Nigeria showed no association between number of teeth lost and education level ${ }^{17}$. Participants with lower level of education in this study may have not received adequate oral health knowledge on preventive behaviors. Furthermore, utilization of oral health services among those who are less educated in most instances is for emergency oral health care rather than restorative care $^{23}$.

In agreement with other studies done in Nigeria ${ }^{17}$ and Brazil ${ }^{24}$ this study also observed that missing teeth in the upper and lower anterior locations was associated with the 35-85 years age group. Observed findings from this study could be due to financial constraints faced by older adults who tend to seek care on a problem-oriented basis ${ }^{25}$. 
Missing teeth in the posterior location of the upper jaw was also common among the older age group in $\mathrm{Ni}$ geria ${ }^{17}$. Other studies done in Tanzania ${ }^{26,27}$ and Ken$\mathrm{ya}^{28}$ found no association between missing teeth in the posterior location of upper jaw and age. The observed findings could be related to the differences in sampling of subjects, however, it could also be related to the tooth anatomy of posterior teeth which predisposes to dental caries.

In this study missing teeth in the upper posterior location and lower anterior location was associated with having primary education or less and these findings corroborate with studies done in Thailand ${ }^{29}$, $\operatorname{Japan}^{30}$ and Brazil $^{31}$. It is possiblthat people with lower level of education lack awareness on prevention of oral diseases and available treatment options.

Although a study done in Nigeria reported that males lost more anterior teeth while females lost more posterior teeth ${ }^{17}$ this study showed no association between location of missing teeth and sex. The possible reason for the lack of association between location of missing teeth and sex could be related to provision of emergency treatment care to all in need regardless of sex.

The results from this study were in agreement with a number of studies ${ }^{6,32}$, whereby the subjective prosthodontic treatment need was associated with having an age of 35 or more years, however, a study done in Norway reported no association between subjective prosthodontic treatment need and age ${ }^{33}$. The cumulative effects of dental diseases can explain the subjective treatment need amongst the older age group.

Unlike a study done in Norway ${ }^{34}$ that reported a higher subjective prosthodontic treatment need in males, a study done in Poland ${ }^{35}$ reported a higher subjective prosthodontic treatment need in females. These findings were contrary to findings reported in this study that showed no association between subjective prosthodontic treatment need and sex. Chewing disability due to missing teeth experienced by both sexes ${ }^{12}$ could explain the lack of difference observed in subjective prosthodontic treatment need between sexes.

While a study done in Brazil reported association between subjective prosthodontic treatment need with higher level of education ${ }^{36}$, this study showed no association between subjective prosthodontic treatment need and education level. It is possible that the subjective prosthetic treatment need of an individual is not influenced by the level of education but rather satisfaction with oral function and aesthetics.

Contrasting to findings from a study in Saudi Arabia ${ }^{5}$ the subjective prosthodontic treatment need of participants in this study who were missing 4 or more teeth was high. The higher subjective prosthodontic treatment need among participants in this study with four or more missing teeth could be related to their reduced oral functionality ${ }^{37}$.

Although it has been reported that the demand for prosthetic replacement is associated with position of the missing teeth ${ }^{38}$ this study reported a higher subjective prosthetic treatment need among participants with missing teeth in the anterior area of the upper jaw, anterior area of the lower jaw and posterior area of the upper jaw. Wide smiles that compromise aesthetics from visibility of edentulous gaps in the posterior area of the upper jaw could have been a contributing factor.

Similar to findings from a study done in Italy ${ }^{39}$, the subjective prosthodontic treatment need of participants in this study was significantly associated with 4 or more missing teeth. The observed subjective prosthodontic treatment need can be due to affected aesthetics and oral function ${ }^{40}$.

Association between subjective prosthodontic treatment need and loss of teeth in the upper anterior location was also reported in Poland ${ }^{41}$. This observed subjective prosthodontic treatment need can be explained by social considerations, compromised aesthetics, speech and/or decreased self-esteem ${ }^{42}$.

\section{Conclusion}

This study highlights that having four or more missing teeth and having tooth loss in the anterior location of the upper jaw were significant contributing factors in expressing patient's subjective prosthodontic treatment need.

\section{Acknowledgement}

Tanzania Ministry of Health, Community Development, Gender, Elderly and Children, Muhimbili University of Health and Allied Sciences, authorities of the dental facilities and participants.

\section{References}

1. Kanno T, Carlsson GE. A review of the shortened dental arch concept focusing on the work by the Käyser/Nijmegen group. J Oral Rehabil. 2006;33(11):850862. doi:10.1111/j.1365-2842.2006.01625.x 
2. Craddock H. Consequences of tooth loss: 1 . The patient perspective--aesthetic and functional implications. Dent Updat. 2009;36(10):616-619. doi:10.12968/ denu.2009.36.10.616

3. Petersen PE, Yamamoto T. Improving the oral health of older people: The approach of the WHO Global Oral Health Programme. Community Dent Oral Epidemiol. 2005;33(2):81-92. doi:10.1111/j.16000528.2004.00219.x

4. Gilbert GH, Paul duncan R, Shelton BJ. Social Determinants of Tooth Loss. Health Serv Res. 2003;38(6p2):1843-1862. doi:10.1111/j.1475-

6773.2003.00205.x

5. Omar R, Tashkandi E, Abduljabbar T, Abdullah MA AR. Sentiments Expressed in Relation to Tooth Loss: A Qualitative Study Among Edentulous Saudis. Int J Prosthodont. 2003;16(5):515-520.

6. McGrath C BR. Can dentures improve the quality of life of those who have experienced considerable tooth loss? J Dent. 2001;29(4):243-46.

7. Elias AC SA. The relationship between satisfaction with mouth and number, position and condition of teeth: studies in Brazilian adults. J Oral Rehabil. 1999;26(1):53-71.

8. Sarita PTN, Witter DJ, Kreulen CM, Matee MI, van't Hof MA, Creugers NHJ. Decayed/missing/filled teeth and shortened dental arches in Tanzanian adults. Int J Prosthodont. 2004;17(2):224-230.

9. Quaker AS. Consequen Ces of Tooth Loss on Oral Function and Need for Rep Lacement of Missing Teeth Among Patients Attending Muhimbili Dental Clinic. Published online 2011.

10. URT. The United republic of Tanzania. National Beaural of Statistics: 2012 Population and Housing Census Population Distribution by Administrative areas. Natl Bur Stat Minist Financ. Published online 2013:177,180.

11. George B, John J, Saravanan S, Arumugham IM. Prevalence of permanent tooth loss among children and adults in a suburban area of Chennai. Indian J Dent Res. 2011;22(2):364. doi:10.4103/0970-9290.84284

12. Bortoluzzi MC, Traebert, Lasta J, Rosa TN Da, Capella DL, Presta AA. Tooth loss, chewing ability and quality of life. Contemp Clin Dent. 2012;3(4):393-397. doi:10.4103/0976-237X.107424

13. Muller F, Naharro M, Carlsson GE. What are the prevalence and incidence of tooth loss in the adult and elderly population in Europe? Clin Oral Implants Res. 2007;18 Suppl 3:2-14. doi:10.1111/j.16000501.2007.01459.x
14. Kutesa A, Mwanika A, Wandera M. Pattern of dental caries in Mulago Dental School clinic, Uganda. Afr Health Sci. 2005;5(1):65-68.

15. Hamasha AA, Sasa I, Al-Qudah M. Risk indicators associated with tooth loss in Jordanian adults. Community Dent Oral Epidemiol. 2000;28(1):67-72.

16. Batista MJ, Rihs LB, Sousa M da LR de. Risk indicators for tooth loss in adult workers. Braz Oral Res. 2012;26(5):390-396.

17. Oremosu O, Uti O. Prevalence of Tooth Loss in A Community in The South-West of Nigeria. J Oral Heal Community Dent. 2014;8(3):154-159.

18. Khalifa N, Allen PF, Abu-bakr NH, Abdel-Rahman ME. Factors associated with tooth loss and prosthodontic status among Sudanese adults. J Oral Sci. 2012;54(4):303-312.

19. Oginni FO. Tooth loss in a sub-urban Nigerian population: causes and pattern of mortality revisited. Int Dent J. 2005;55(1):17-23.

20. Okullo I, Astrøm AN, Haugejorden O, Rwenyoni C. Variation in caries experience and sugar intake among secondary school students in urban and rural Uganda. Acta Odontol Scand. 2003;61(4):197-202.

21. Jiang Y, Okoro CA, Oh J, Fuller DL. Sociodemographic and health-related risk factors associated with tooth loss among adults in rhode island. Prev Chronic Dis. 2013;10:E45.

22. Perera R, Ekanayake L. Tooth loss in Sri Lankan adults. Int Dent J. 2011;61(1):7-11. doi:10.1111/j.1875595X.2011.00002.x

23. Kikwilu EN, Masalu JR, Kahabuka FK, Senkoro AR. Prevalence of oral pain and barriers to use of emergency oral care facilities among adult Tanzanians. BMC Oral Health. 2008;8(1):1-7. doi:10.1186/1472-6831-8-28 24. Montandon A, Zuza E, Toledo BE. Prevalence and reasons for tooth loss in a sample from a dental clinic in Brazil. Int J Dent. Published online 2012.

25. Gilbert GH, Duncan RP, Shelton BJ. Social Determinants of Tooth Loss. Health Serv Res. 2003;38(6 II):1843-1862. doi:10.1111/j.1475-6773.2003.00205.x

26. Mumghamba EGS, Fabian FM. Tooth loss among habitual chewing-stick and plastic toothbrush users in the adult population of Mtwara, rural Tanzania. Int J Dent Hyg. 2005;3(2):64-69. doi:10.1111/j.16015037.2005.00131.x

27. Kida IA, Astrøm AN, Strand G V, Masalu JR. Clinical and socio-behavioral correlates of tooth loss: a study of older adults in Tanzania. BMC Oral Health. 2006;6(5):1-10.

28. Sanya BO, Ng'ang'a PM, Ng'ang'a RN. Causes and 
pattern of missing permanent teeth among Kenyans. East Afr Med J. 2004;81(6):322-325.

29. Chatrchaiwiwatana S, Ratanasiri A, Jaidee J, Soontorn S. Factors related to tooth loss due to dental caries among workers in an industrial estates in Thailand. $J$ Med Assoc Thai. 2012;95 Suppl 1:S1-6.

30. Ando A, Ohsawa M, Yaegashi Y, et al. Factors related to tooth loss among community-dwelling middle-aged and elderly Japanese men. J Epidemiol. 2013;23(4):301306. doi:10.2188/jea.JE20120180

31. Barbato PR, Muller Nagano HC, Zanchet FN, Boing AF, Peres MA. [Tooth loss and associated socioeconomic, demographic, and dental-care factors in Brazilian adults: an analysis of the Brazilian Oral Health Survey, 2002-2003]. Cad Saude Publica. 2007;23(8):18031814.

32. Fraczak B, Lupa-Buhmann J, Sobolewska E. [Prosthetic treatment needs among the elder in Germany]. Ann Acad Med Stetin. 2007;53(3):134-139.

33. Trovik TA, Klock KS HO. Predictors of norwegian adult patients' perceived need for replacement of teeth at the time of extraction. Community Dent Heal. 2002;19(2):79-85.

34. Trovik TA, Klock KS, Haugejorden O. Level and predictors of agreement between patients and their dentists concerning need for replacement of teeth at the time of extraction. Acta Odontol Scand. 2002;60(3):186192.

35. Nyyssonen V, Lappalainen R, Honkala E, Markkanen H, Paunio I. Subjective need of removable denture treatment in Finnish adults. I Oral Rehabil. 1987;14(4):393-398.

36. Roncalli AG, Tsakos G, Sheiham A, de Souza GC, Watt RG. Social determinants of dental treatment needs in Brazilian adults. BMC Public Health. 2014;14:1097. doi:10.1186/1471-2458-14-1097

37. Nikolovska J, Korunoska-Stevkovska V, Mijoska A, Popovska L. Prosthodontics status and treatment needs among the elderly in the Republic of Macedonia. Open Access Maced J Med Sci. 2018;6(5):874-878. doi:10.3889/ oamjms.2018.190

38. Leles CR, Freire M do CM. A sociodental approach in prosthodontic treatment decision making. $J$ Appl Oral Sci. 2004;12(2):127-132. doi:10.1590/s167877572004000200009

39. Melilli D, Matranga D, Cassaro A, Pizzo G. [Edentulousness and prosthetic treatment needs in a sample of the adult inhabitants of Palermo (Italy)]. Ann Ig. 2010;22(1):69-81.

40. Akeel R. Attitudes of Saudi male patients toward the replacement of teeth. J Prosthet Dent. 2003;90(6):571577. doi:10.1016/S0022391303006231

41. Mielnik-Blaszczak M, Stodolkiewicz M, Krawczyk D, Borowska M, Kleinrok J. Evaluation of the masticatory system condition with respect to the need for prosthetic treatment in the population of 35-44-yearolds from the Lublin Region. Ann Univ Mariae Curie Sklodowska Med. 2004;59(1):204-208.

42. Walter MH, Wolf $\mathrm{BH}$, Rieger C, Boening KW. Prosthetic treatment need in a representative German sample. J Oral Rehabil. 2001;28(8):708-716. 